\title{
Assessing local risk factors of Beijing Hand-Foot- Mouth Disease in China
}

\author{
Jiaojiao Wang ${ }^{\star 1}$, zhidong cao', Daniel D. Zeng ${ }^{1,2}$ and Quanyi Wang ${ }^{3}$ \\ ${ }^{1}$ The State Key Laboratory of Management and Control for Complex Systems, Institute of Automation, Chinese Academy of Sciences, \\ Beijing, China; ${ }^{2}$ University of Chinese Academy of Sciences, Beijing, China; ${ }^{3}$ Beijing Center for Disease Prevention and Control \\ (CDC), Beijing, China
}

\section{Objective}

HFMD incidence varies between geographic regions at the township in Beijing. The objective of this study was to examine spatial heterogeneity for the association between HFMD incidence and demographic and socioeconomic factors.

\section{Introduction}

Hand-foot-mouth disease (HFMD) is a common childhood illness and the drivers of HFMD incidence are still not clear [1]. In mainland China, continuing and increasing HFMD epidemics have been recorded since 2008, causing millions of infections and hundreds of deaths annually. In Beijing, 28,667 cases were reported in 2015 and the incidence was 133.28/100,000. The variations in Beijing HFMD epidemics over population, space, and time that have been revealed [2] emphasize the need for further research about risk factors of HFMD occurrence. This study aims to explore local effects on HFMD incidence led by potential factors.

\section{Methods}

HFMD Data. Beijing HFMD data during 2008-2012 period were provided by the Beijing Center for Disease Prevention and Control. HFMD incidence adopted in this study was the annual average value during the five years.

Predictor variables. Potential risk factors obtained from the case records (demographic, occupation, health-seeking behavior) and spatial POIs (points of interest) consisted of 22 variables involving residence, restaurant, education, medical facilities, business facilities, infrastructure. The scale of different kinds of POIs $(1 / 100,000)$ was noted by calculating the ratio of the number of POIs to the population at certain township or street committee.

Model Specification. Some initial associations between HFMD incidence and 8 predictor variables (population density, shopping mall, supermarket, pharmacy, kindergarten, middle school, parking lot, health seeking behavior) were revealed using Pearson correlation analysis and the exploratory regression. An ordinary least squares (OLS) model was fitted to diagnose the residual normality and dependence. Geographically weighted regression (GWR) was chosen to model the relationship, compare the difference from OLS regression and measure how much improvement the local model gained.

\section{Results}

GWR model with residual independence (Moran's I $=0.0214$, $\mathrm{p}=0.3405)$ and lower AICc, performing much better than OLS model with residual dependence (Moran's $\mathrm{I}=0.1271, \mathrm{p}=0.0000$ ) and higher AICc. Prediction accuracy by GWR (local $\mathrm{R}^{2}$ ranging from 0.42 to $\left.0.90, \mathrm{R}^{2}=0.88\right)$ was higher than that by OLS $\left(\mathrm{R}^{2}=0.57\right)$. The higher local $\mathrm{R}^{2}$ values clustered in the east of Fangshan and Urban-Rural Transition Area. Higher coefficient for intercept mainly occurred in north-western and south-eastern portion of Beijing. The coefficients for predictors showed shifting patterns from positive to negative at different township. The local effects led by supermarket and shopping mall showed similar spatial pattern, as well as those led by kindergarten and middle school. The scale of pharmacy was positively related to HFMD incidence in the west of Daxing and the junction part of Chaoyang and Tongzhou.

\section{Conclusions}

This study quantitatively assessed local risk factors of Beijing HFMD occurred in China using GWR model which outperformed OLS regression. The findings could provide valuable information for adequate disease intervention measures and regional policy.
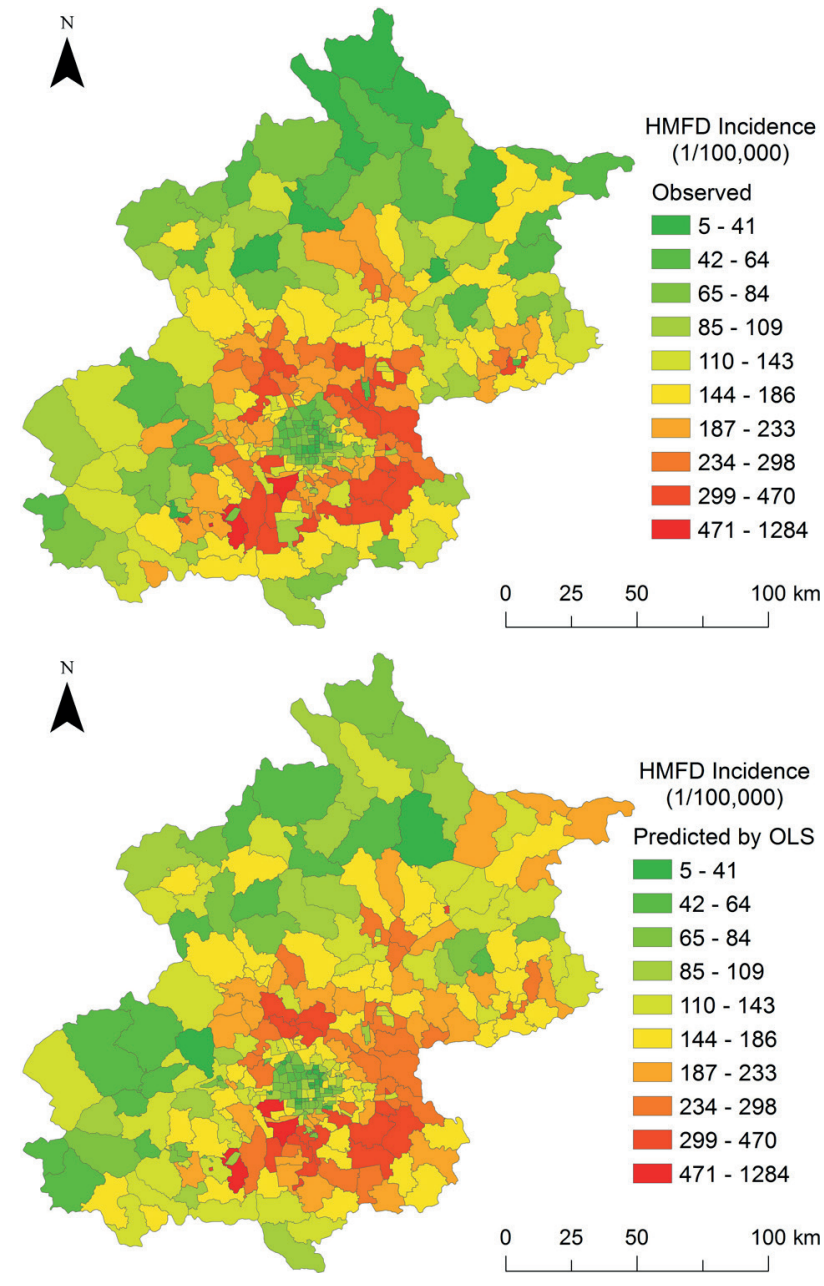

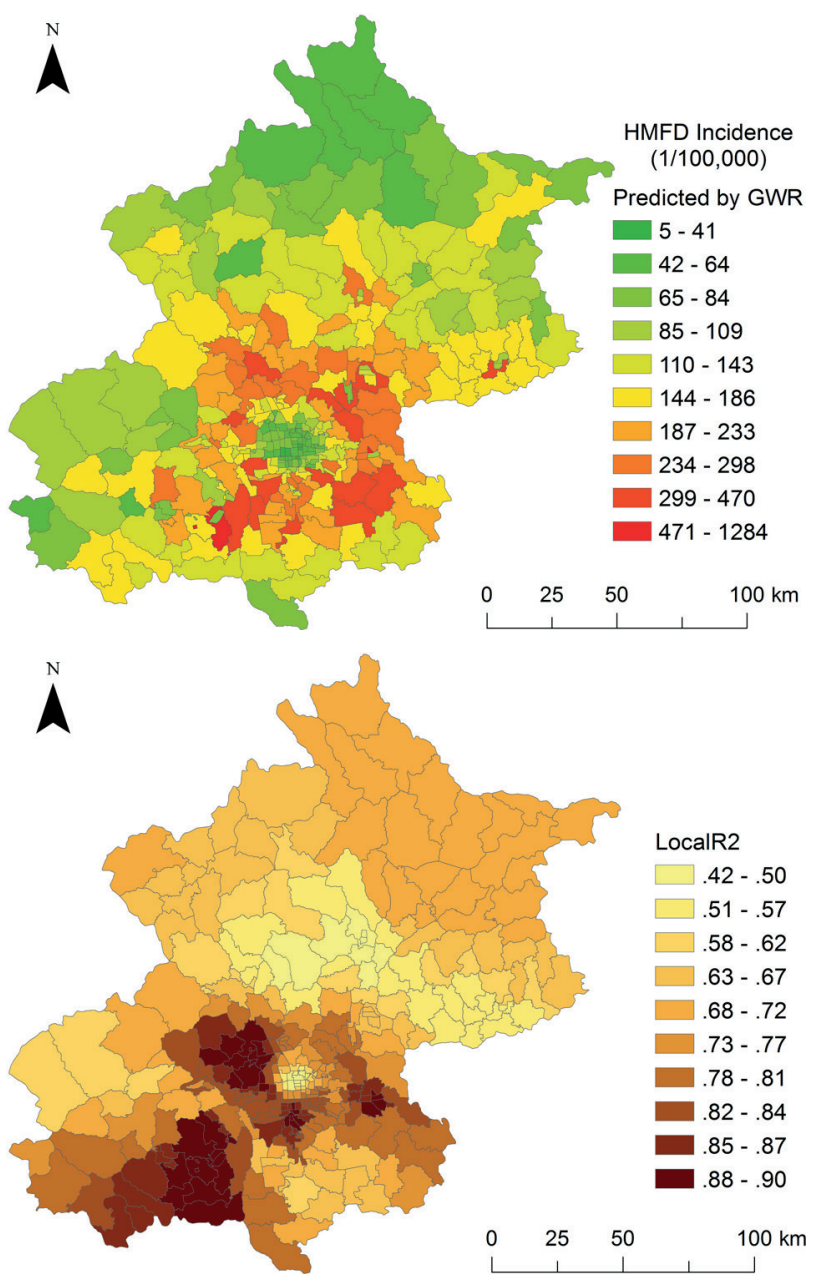

\section{Keywords}

Demographic and socioeconomic factors; Spatial heterogeneity; Geographically weighted regression

\section{Acknowledgments}

Supported by NSFC (71603253), the National Major Research Program of China (2016YFC1200702) and the Early Career Development Award of SKLMCCS (Y3S9021F37).

\section{References}

1. Lu J, Sun L, Zeng H, et al. Enterovirus Contamination in Pediatric Hospitals: A Neglected Part of the Hand-Foot-Mouth Disease Transmission Chain in China?. Clinical Infectious Diseases, 2016, 62(4): 524-525.

2. Wang J, Cao Z, Zeng D D, et al. Epidemiological analysis, detection, and comparison of space-time patterns of beijing hand-foot-mouth disease (2008-2012). PLoS one, 2014, 9(3): e92745.

\section{*Jiaojiao Wang}

E-mail: jiaojiao.wang@ia.ac.cn 